\title{
Letter Regarding Levosimendan in a Rat Model of Severe Verapamil Poisoning
}

Dear Editor,

I read with interest the original research article describing the use of levosimendan in a rat model of severe verapamil poisoning in the March 2009 issue of the Journal of Medical Toxicology [1]. A number of issues are apparent with this study. First, the authors state: "Although there are no formal studies on the subject, the advent of a class of $\mathrm{Ca}^{2+}$ sensitizers was thought to afford a novel approach to CCB poisoning." The statement regarding the absence of formal studies with levosimendan in verapamil poisoning is incorrect. We have reported 3 separate animal studies to date investigating the effects of levosimendan in severe verapamil poisoning [2-4]. The hemodynamic effects of levosimendan have been compared with calcium chloride, norepinephrine, and 4-aminopyridine in severe verapamil poisoning. In all 3 studies heart rate, continuous arterial BP, and cardiac output were monitored. Levosimendan alone produces moderate improvements in cardiac output but not arterial BP compared with the other treatments. When levosimendan is coadministered with each of the other treatments, there is only minimal improvement in BP from that seen at peak verapamil toxicity. Second, Abraham et al note that levosimendan is not only a myocardial calcium sensitizer but also a mild phosphodiesterase inhibitor. They fail to mention that it is also a peripheral vascular potassium channel agonist, producing vasodilatation and reduced afterload, an effect that may be beneficial in some forms of heart failure [5]. We theorize that the $\mathrm{K}^{+}$-channel agonist properties of this agent augment the vasodilatory properties of verapamil and may not be reversible with the addition of other vasoconstrictors. Third, the authors administered levosimendan as 2 relatively large loading doses but also state they cannot guarantee the concentration of the drug used due to difficulty in dissolution of the powdered compound. Levosimendan is commonly administered as a loading dose and lower-dose infusion [6]. The use of 2 bolus doses does not parallel clinical practice with this agent. The second, larger bolus dose may have accelerated the demise of the animals because of significant vasodilation resulting from suddenly increasing levosimendan concentrations. Finally, using death as the major outcome is a very crude measure of drug effect. The authors should have considered recording either invasive arterial blood pressure or at least noninvasive tail cuff blood pressure to better define the effects of levosimendan in verapamil poisoning.

Andis Graudins, MBBS, PhD

Director, Clinical and Experimental Toxicology Unit,

Department of Emergency Medicine

Prince of Wales Hospital, Barker Street, Randwick, NSW, 2031, Australia

School of Medical Sciences, Faculty of Medicine, University of New South Wales, Kensington, NSW, Australia.

Email: andis.graudins@unsw.edu.au

Dr. Graudins' research has been supported by a University of NSW Facutly research grant and ACMT-Orphan Medical Research Grand. Levosimendal used in this research was donated by Abbott Australasia.

\section{REFERENCES}

1. Abraham MK, Scott SB, Meltzer A, et al. Levosimendan does not improve survival time in a rat model of verapamil toxicity. J Med Toxicol 2009;5(1):3-7.

2. Graudins A, Najafi JM, Perry M. Treatment of experimental verapamil poisoning with levosimendan utilizing a rodent model of drug toxicity. Clin Toxicol 2008;46(1):50-56.

3. Ka Ling K, Graudins A. Treatment of experimental verapamil poisoning with levosimendan and 4-aminopyridine (abstract). Clin Toxicol 2008;46(7):593.

4. Najafi JM, Graudins A. Norepinephrine co-administered with levosimendan does not reverse hypotension in a rodent model experimental verapamil poisoning (abstract). Emerg Med Australas 2007;19(Suppl1):A19-A20.

5. Hohn J, Pataricza J, Petri A, et al. Levosimendan interacts with potassium channel blockers in human saphenous veins. Basic Clin Pharmacol Toxicol 2004;94(6):271-273.

6. Huang L, Weil MH, Tang W, et al. Comparison between dobutamine and levosimendan for management of postresuscitation myocardial dysfunction. Crit Care Med 2005;33(3): 487-491. 